International Journal of Bifurcation and Chaos, Vol. 12, No. 8 (2002) 1859-1868

(c) World Scientific Publishing Company

\title{
CYCLING CHAOS IN ONE-DIMENSIONAL COUPLED ITERATED MAPS
}

\author{
ANTONIO PALACIOS \\ Department of Mathematics, San Diego State University, \\ San Diego, CA 92182-7720, USA
}

Received July 11, 2001; Revised August 3, 2001

\begin{abstract}
Cycling behavior involving steady-states and periodic solutions is known to be a generic feature of continuous dynamical systems with symmetry. Using Chua's circuit equations and Lorenz equations, Dellnitz et al. [1995] showed that "cycling chaos", in which solution trajectories cycle around symmetrically related chaotic sets, can also be found generically in coupled cell systems of differential equations with symmetry. In this work, we use numerical simulations to demonstrate that cycling chaos also occurs in discrete dynamical systems modeled by onedimensional maps. Using the cubic map $f(x, \lambda)=\lambda x-x^{3}$ and the standard logistic map, we show that coupled iterated maps can exhibit cycles connecting fixed points with fixed points and periodic orbits with periodic orbits, where the period can be arbitrarily high. As in the case of coupled cell systems of differential equations, we show that cycling behavior can also be a feature of the global dynamics of coupled iterated maps, which exists independently of the internal dynamics of each map.
\end{abstract}

Keywords: Coupled cell systems; cycling chaos; symmetry.

\section{Introduction}

Symmetry in nonlinear dynamical systems can force certain subspaces of the phase-space to be invariant under the governing equations. In continuous systems, where the governing equations typically consist of systems of differential equations, it is well-known that the presence of invariant subspaces can lead to the existence of solution trajectories that connect, via saddle-sink connections, equilibria and or/periodic solutions that lie on the invariant subspaces. As time evolves, a typical trajectory stays for increasingly longer periods near each solution before it makes a rapid excursion to the next solution. See, for example, [Field, 1980] and [Guckenheimer \& Holmes, 1988]. Since saddlesink connections are robust, these cycles - called heteroclinic cycles — are robust under perturbations that preserve the symmetry of the system.
When a heteroclinic cycle is also asymptotically stable, it serves as a model for a certain kind of intermittency, since nearby trajectories move quickly between solutions (equilibria and periodic solutions) and stay for a relatively long time near each solution. In systems of differential equations with O(2) symmetry, Armbruster et al. [1988] showed that heteroclinic cycles between steady-states can occur stably, and Melbourne et al. [1989] provided a method for finding cycles that involve steady-states as well as periodic solutions. Buono et al. [2000] showed that cycles connecting steady-states and periodic solutions are also found stably in systems of coupled identical cells with Dihedral $\mathbf{D}_{n}$ symmetry.

In principle, more complex heteroclinic cycles can also occur robustly if the invariant subspaces contain more complicated solutions. For instance, replacing the equilibria in the 
Guckenheimer-Holmes cycle with chaotic attractors can lead to what Dellnitz et al. [1995] call cycling chaos. This can be done as follows. First, Dionne et al. [1996] showed that the GuckenheimerHolmes system can be interpreted, with an appropriate choice of coupling function, as a network of identical coupled cells modeled by a system of differential equations of the form

$$
\frac{d X_{i}}{d t}=f\left(X_{i}\right)+\sum_{j \rightarrow i} \alpha_{i j} h\left(X_{i}, X_{j}\right)
$$

where $X_{i}=\left(x_{i 1}, \ldots, x_{i k}\right) \in \mathbf{R}^{k}$ denotes the state variables of cell $i, f$ governs the internal dynamics of each cell, $h$ is the coupling function between two cells, the summation is taken over those cells $j$ that are coupled to cell $i$, and $\alpha_{i j}$ is a matrix of coupling strengths. Dellnitz et al. [1995] then made the critical observation that, under certain conditions, cycling behavior is a feature of the global dynamics that can persist independently of the internal dynamics of each cell. It follows that if the internal dynamics of the Guckenheimer-Holmes system is replaced by a dynamical system set to produce a chaotic attractor, then the new coupled system would produce cycling chaos. Dellnitz et al. demonstrated this conclusion using first Chua's circuit and then Lorenz equations.

Although the existence and stability of heteroclinic cycles in continuous dynamical systems with symmetry has been extensively explored, little is known, however, about the existence of these cycles in discrete systems. In this work, we explore numerically the existence of heteroclinic cycles in coupled cell maps with internal cell dynamics modeled by one-dimensional iterative maps. We demonstrate that discrete systems can also exhibit cycles connecting fixed points with fixed points and periodic orbits with periodic orbits, where the period can be arbitrarily high. More importantly, we show that cycling behavior can persist, independently of the internal dynamics of each cell, as a feature of the global dynamics. A detailed analysis of existence and stability of these cycles is in preparation [Palacios, 2002].

\section{Coupled Iterated Cell Maps}

We consider here similar coupled cell systems to those used by Dellnitz et al. [1995] for showing the existence of cycling chaos, except that now we assume the internal dynamics of each cell is governed by one-dimensional maps of the form

$$
x_{i_{n+1}}=f\left(x_{i_{n}}, \lambda\right)
$$

where $x_{i_{n}} \in \mathbf{R}$ denotes the state variable of cell $i$ and $\lambda \in \mathbf{R}$ is a parameter. We model an interconnected network of $N$ identical cells, by a system of one-dimensional maps of the form

$$
x_{i_{n+1}}=f\left(x_{i_{n}}, \lambda\right)+\sum_{j \rightarrow i} \alpha_{i j} h\left(x_{i_{n}}, x_{j_{n}}\right)
$$

where $h$ is the coupling function between those cells $j$ that are coupled to cell $i, 1 \leq i \leq N$. Following Dellnitz et al. [1995], we use the term local symmetries to refer to the symmetries of individual cells. Thus, $\Sigma$ is a group of local symmetries if, for all $\sigma \in \Sigma$, we have

$$
f(\sigma x)=\sigma f(x) .
$$

Similarly, we use the term global symmetries to refer to the symmetries that are induced by the pattern of coupling. We also consider wreath product coupling, so that the local symmetries of individual cells are also symmetries of the network equations (3). That is, if $\sigma \in \Sigma$ then

$$
\begin{aligned}
& h\left(x_{i}, \sigma x_{j}\right)=\sigma h\left(x_{i}, x_{j}\right), \\
& h\left(\sigma x_{i}, x_{j}\right)=h\left(x_{i}, x_{j}\right) .
\end{aligned}
$$

For illustrative purposes, in this work, we consider systems of only three identical cells coupled in a directed ring as is shown schematically in the following diagram. Similar ideas can be applied to systems with larger number of cells.

Assuming the coupling among cells is also identical, $\alpha_{i j}=\alpha$, we write the network equations in the form

$$
\begin{aligned}
& x_{n+1}=f\left(x_{n}, \lambda\right)+\alpha h\left(y_{n}, x_{n}\right) \\
& y_{n+1}=f\left(y_{n}, \lambda\right)+\alpha h\left(z_{n}, y_{n}\right) \\
& z_{n+1}=f\left(z_{n}, \lambda\right)+\alpha h\left(x_{n}, z_{n}\right)
\end{aligned}
$$

so that the global symmetries of the network are described by the cyclic group $\mathbf{Z}_{3}$, which is generated by the permutation

$$
(x, y, z) \mapsto(y, z, x)
$$




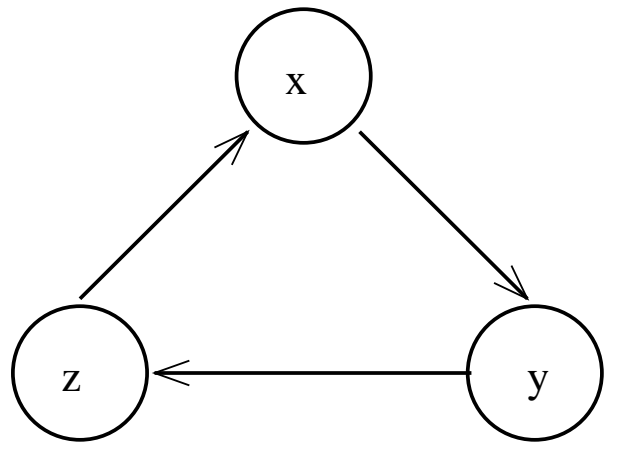

Fig. 1. $\mathbf{Z}_{3}$-symmetric directed ring with three identical cells.

\section{Results of Simulations}

As a first example, we assume the internal dynamics of each cell is governed by a cubic map with local $\mathbf{Z}_{2}$ symmetry through

$$
f(x, \lambda)=\lambda x-x^{3}, \quad \lambda>0 .
$$

For different values of the bifurcation parameter $\lambda$, this map exhibits period-doubling cascades leading to chaotic attractors. See the bifurcation diagram of Fig. 2.

The bifurcations in (5) are reminiscent of those found in the logistic map [May, 1976], except that now local $\mathbf{Z}_{2}$-symmetry forces two nontrivial fixed points (one with $x>0$ and one with $x<0$ ) to bifurcate from the trivial solution $x=0$ at $\lambda=1$. Each fixed point, in turn, undergoes a period-doubling cascade leading to a pair of chaotic attractors. Local $\mathbf{Z}_{2}$ symmetry again forces the cascades to occur at the same parameter values for each fixed point [Dellnitz et al., 1995]. For $\lambda<\lambda_{c}=3 \sqrt{3} / 2$, the attractors are confined to opposite sides of the $x=0$ axis and each attractor has its own basin of attraction. At $\lambda=\lambda_{c}$, the basins of attraction collide and the two attractors merge into a single one. Rogers and Whitley [1983] provided a more comprehensive analysis of a similar map $f(x, \lambda)=-\left(\lambda x-x^{3}\right)$, $\lambda>0$.

Next we form the interconnected network equations (4) with coupling function

$$
h\left(x_{i}, x_{j}\right)=\left|x_{j}\right|^{1 / 2} x_{i}
$$

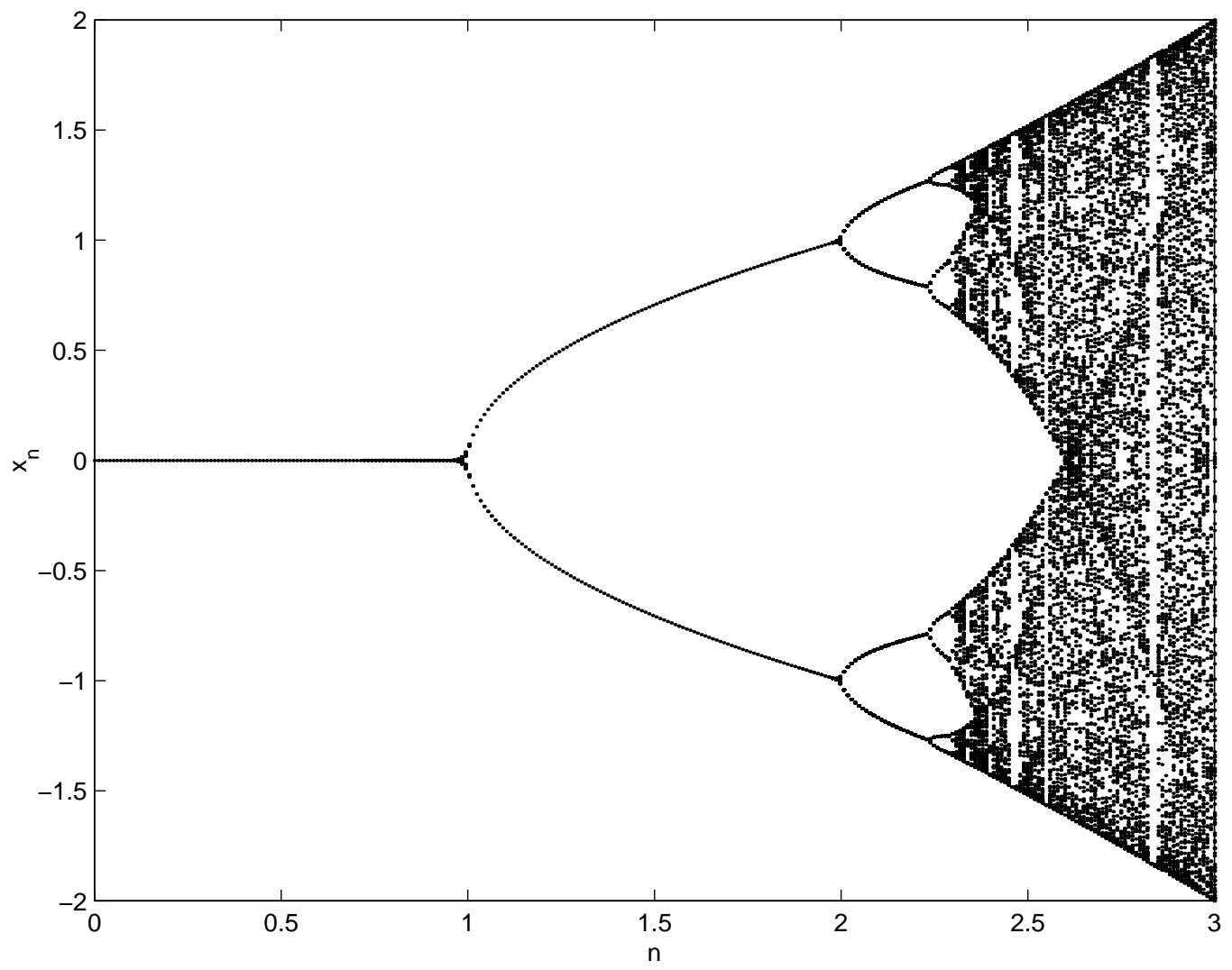

Fig. 2. Bifurcation diagram for a cell with internal dynamics $f(x, \lambda)=\lambda x-x^{3}$. 

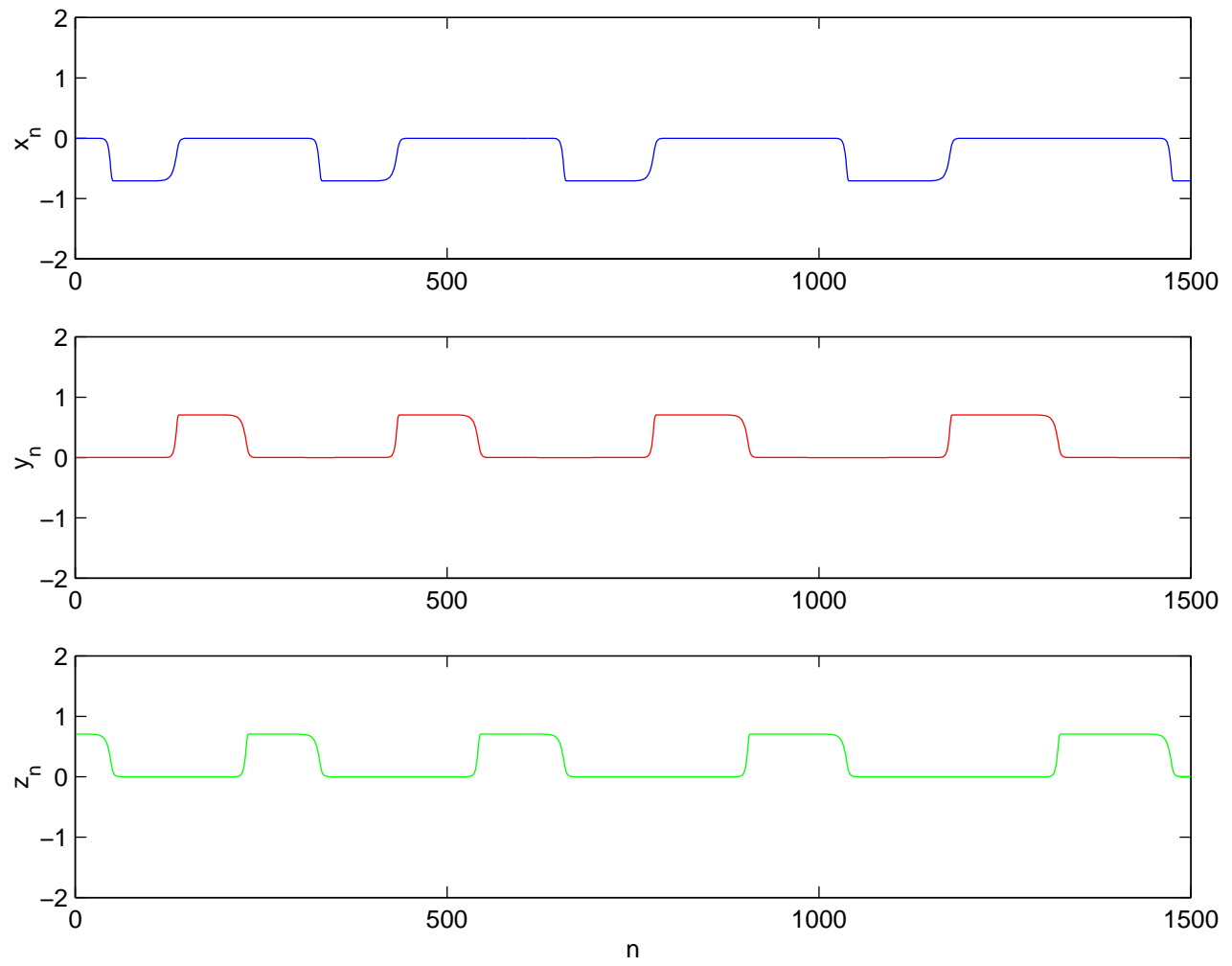

(a)
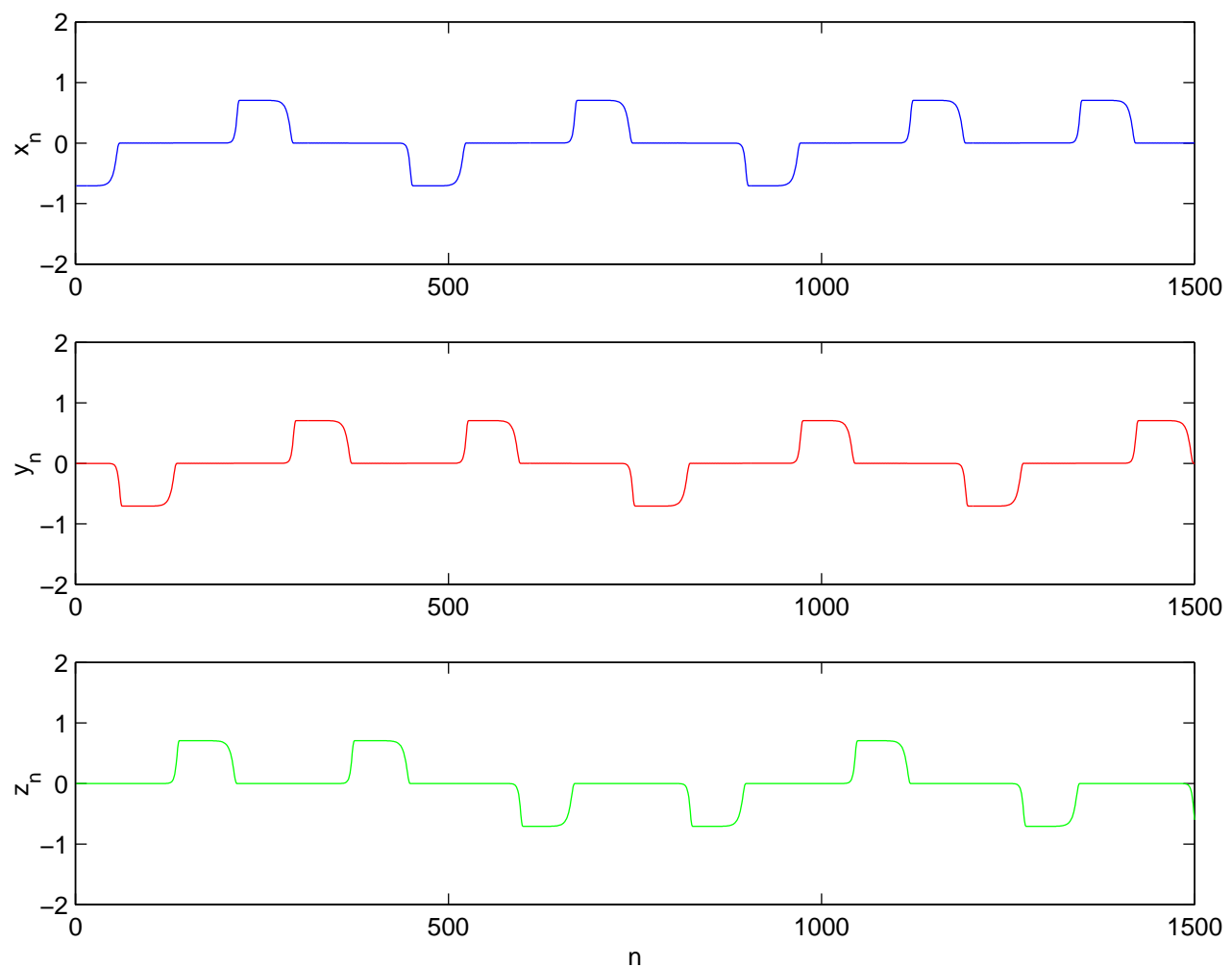

(b)

Fig. 3. Two types of cycles connecting fixed points of $f(x, \lambda)=\lambda x-x^{3}$ at $\lambda=1.5$. (a) Type I: With coupling strength $\alpha=-1$, an active cell always shows the same of two conjugate solutions. (b) Type II: With a different coupling strength $\alpha=-2.795$, each active cell switches between two conjugate solutions. 
so that the three-cells coupled system possesses global $\mathbf{Z}_{3}$-symmetry and local $\mathbf{Z}_{2}$-symmetry. Using an appropriate value of the coupling strength $\alpha$ and a fixed value of $\lambda$ in the interval $1 \leq \lambda \leq \lambda_{c}$, the coupled cell system (4) exhibits trajectories that cycle around the orbits generated by the internal dynamics of each cell at each value of $\lambda$. The wide range of behavior in the cubic map (5), which controls the internal dynamics of individual cells, leads to cycles connecting fixed points with fixed points, periodic orbits with periodic orbits, and chaotic attractors with chaotic attractors. A typical trajectory in these cycles produces a pattern in which, at any given time, one of the cells is active (in a fixed point, periodic orbit or chaotic orbit) while the remaining two are quiescent. Depending on the coupling strength, two types of cycling patterns are observed. With relatively weak coupling (in absolute value), we find a cycle (type I) in which when a cell becomes active it always selects the same of the two conjugate orbits of Fig. 2, either $x>0$ or $x<0$. The actual orbit that is selected depends on the initial conditions of the active cell. An example of such cycle connecting fixed points of (2) is illustrated in Fig. 3. All subsequent figures related to the first example were created using the initial conditions $\left(x_{0}, y_{0}, z_{0}\right)=(-0.01,0.03,0.02)$. With stronger coupling (in absolute value), we now find a different type of cycle (type II) in which each cell switches intermittently between conjugate orbits as is also shown in Fig. 3 .

As $\lambda$ increases towards $\lambda_{c}$, both cycles of Fig. 3 persist as a feature of the global dynamics of the coupled map system. The orbits visited by the cycles, however, change as the internal dynamics of each map changes according to the bifurcation diagram of Fig. 2. For instance, at $\lambda=2$ the fixed points of the cycle of Fig. 3 undergo a period-doubling bifurcation that leads to two conjugate stable period-two orbits. Near this bifurcation point, we again find two types of cycles: one connecting the same of two conjugate period-two orbits and one where switching between conjugate period-two orbits occurs intermittently. An example of the former type of cycle is shown in Fig. 4. As the period-doubling cascade in the internal
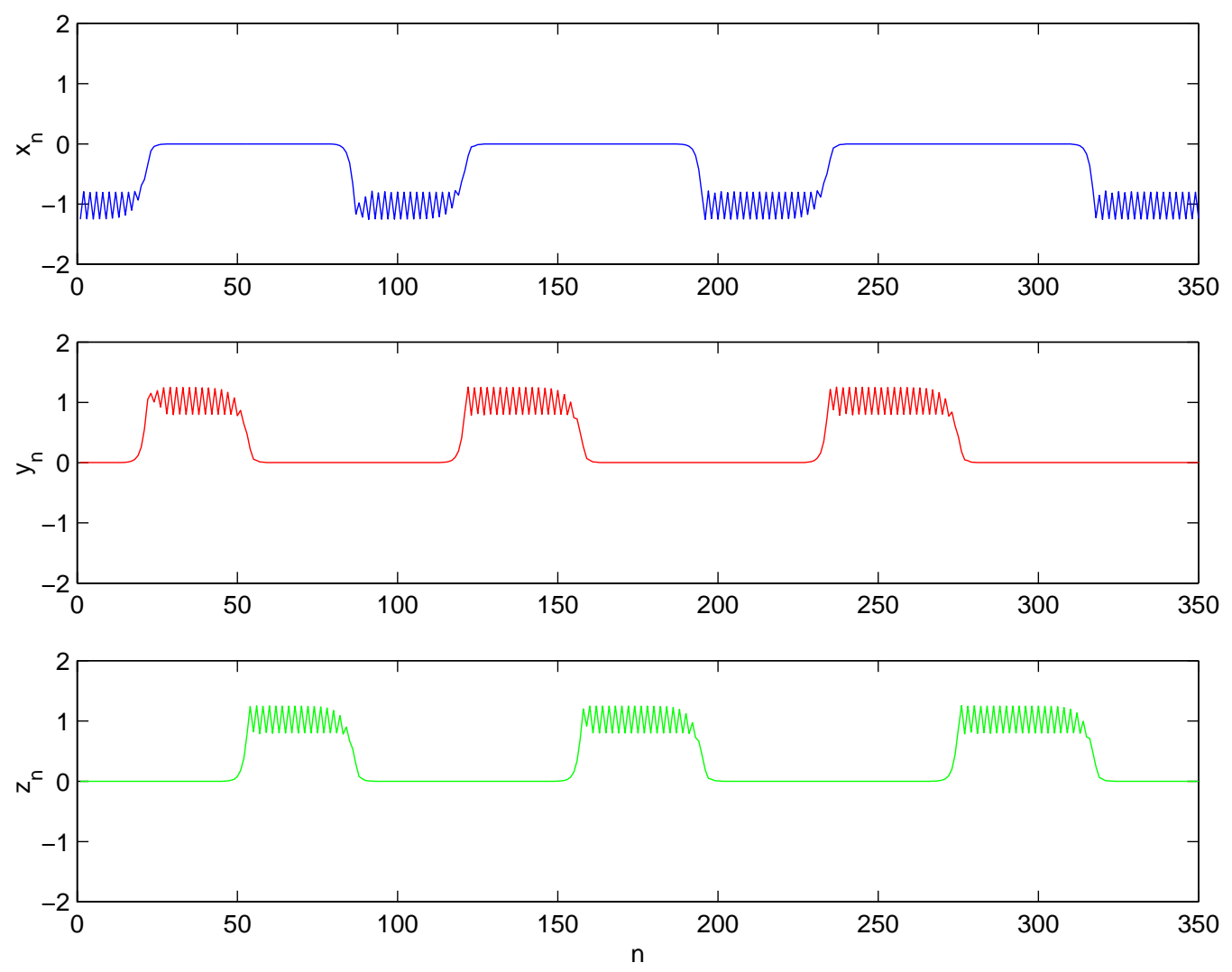

Fig. 4. Type I heteroclinic cycle connecting period-two orbits of $f(x, \lambda)=\lambda x-x^{3}$ at $\lambda=2.2$ and coupling strength $\alpha=$ -1.7 . 

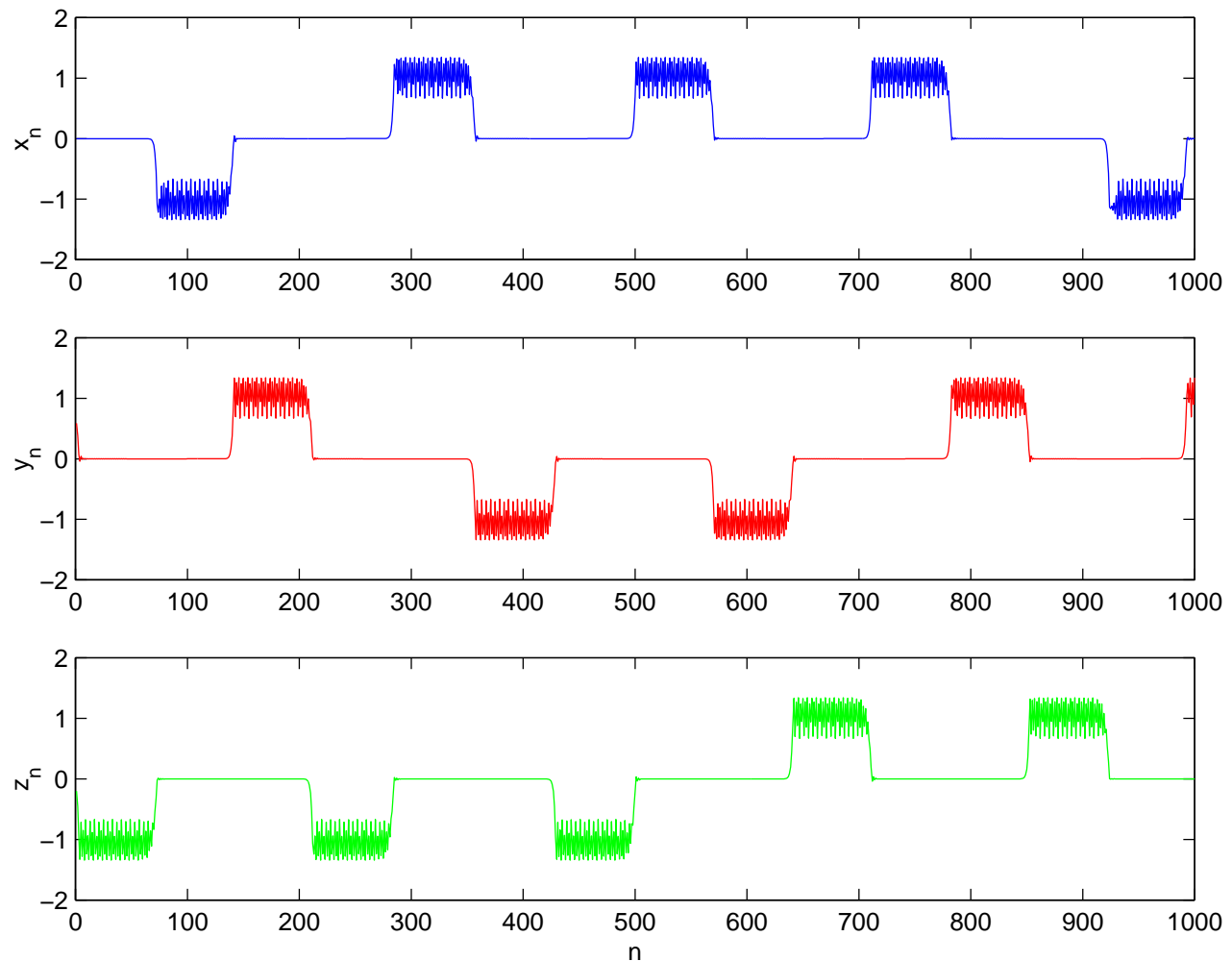

(a)
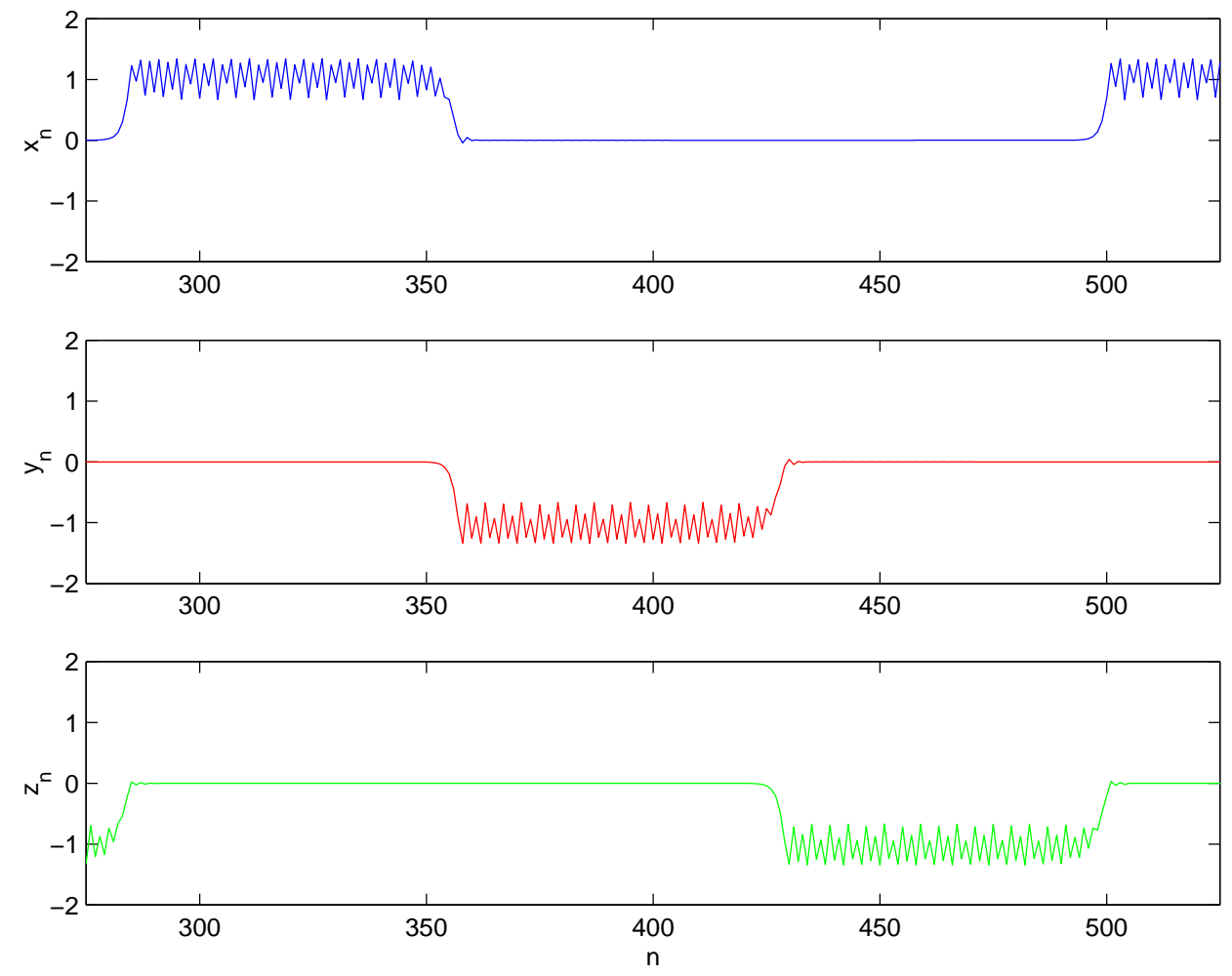

(b)

Fig. 5. (a) Type II heteroclinic cycle connecting period-four orbits of $f(x, \lambda)=\lambda x-x^{3}$ at $\lambda=2.3$ and coupling strength $\alpha=-2.91$. (b) Magnification of cycle shown in (a). 

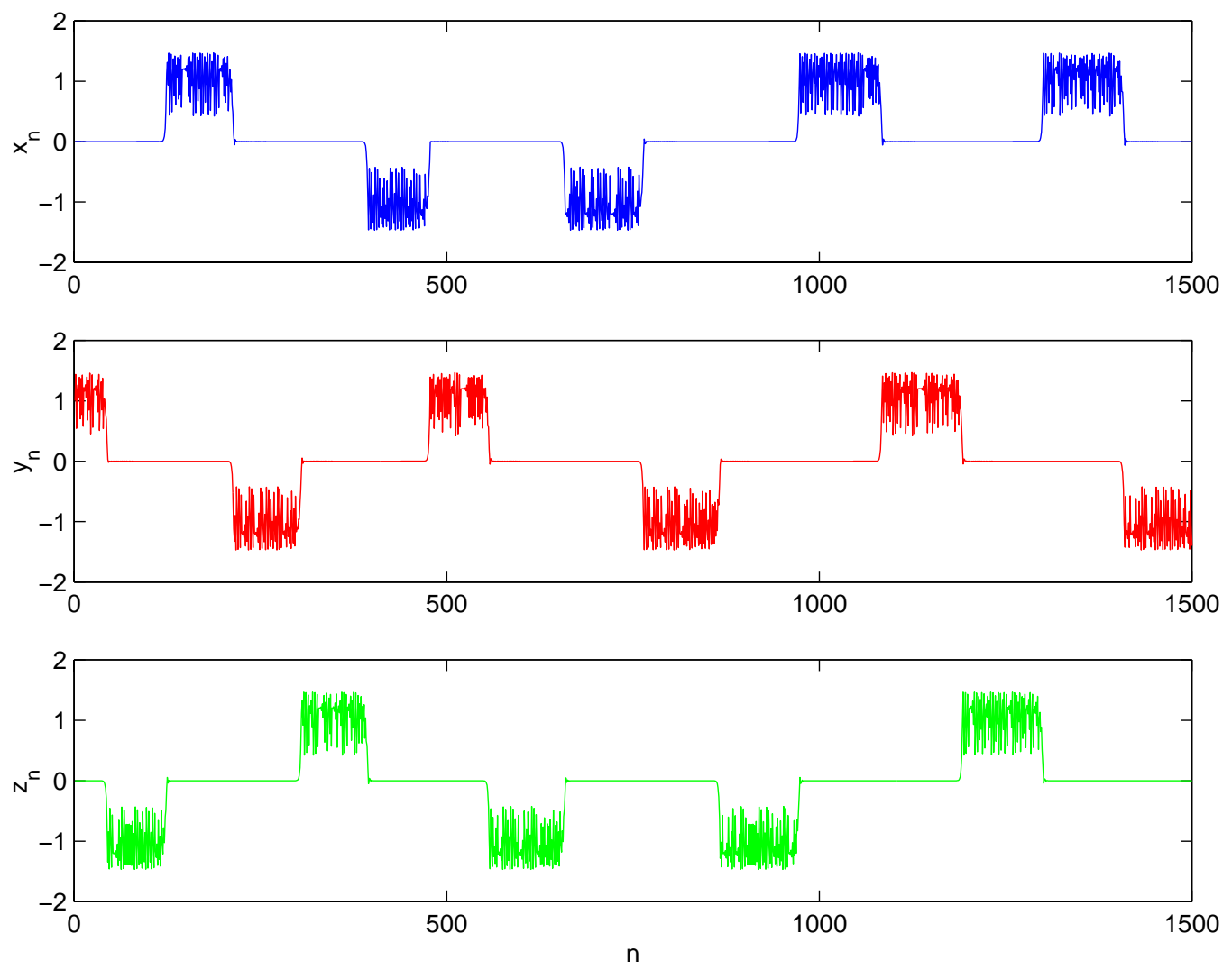

Fig. 6. Cycling chaos connecting chaotic attractors of the coupled cell system (4) with $f(x, \lambda)=\lambda x-x^{3}$, at $\lambda=2.44$ and coupling strength $\alpha=-2.71$.

dynamics of each cell continues, cycles involving periodic orbits of higher period also occur robustly. A representative example with random switching between conjugate period-four orbits is shown in Fig. 5.

The cycling behavior illustrated thus far is also found at values of the bifurcation parameter (within $1 \leq \lambda \leq \lambda_{c}$ ) where the attractor is a chaotic orbit. For instance, at $\lambda=2.44$ calculations of Lyapunov exponents (not shown for brevity) confirm the existence of an asymmetric chaotic attractor filling parts of the interval $[0,2]$. Local $\mathbf{Z}_{2}$ symmetry in the internal dynamics of each cell forces the existence of a conjugate chaotic attractor within $[-2,0]$. Depending on the coupling strength, we also find at $\lambda=2.44$ two types of cycles connecting chaotic orbits. With $\alpha=-2.7$, for instance, we can observe in Fig. 6 cycling chaos that is qualitatively similar to the one found by Dellnitz et al. [1995] using Chua's continuous circuit model. That is, an active cell switches randomly between two conjugate chaotic orbits. With a different value of coupling strength $\alpha=-1.9$, the cycling chaos persists but active cells can no longer switch between conjugate chaotic orbits. This latter type of cycle (not shown for brevity) does not seem to appear in [Dellnitz et al., 1995].

At $\lambda=\lambda_{c}$, the basins of attraction of the two chaotic attractors collide and the two attractors merge into one. For values of $\lambda$ slightly greater than $\lambda_{c}$, Manneville [1990] showed that the internal dynamics of each map, in the uncoupled system, produces an intermittent orbit that switches between the remnants of the two attractors. When the cells are coupled, we obtain a third type of cycling chaos (see Fig. 7) in which switching between the two remnants of the attractors occurs during the interval of activity of each cell.

In our second and last example, we assume the internal dynamics of each cell to be governed by the standard logistic map

$$
f(x, \lambda)=\lambda x(1-x), \quad \lambda>0,
$$

so that the local symmetry is now determined by the identity group $\mathbf{1}$. We use a coupling function of the form

$$
h\left(x_{i}, x_{j}\right)=\left|x_{j}\right|^{1 / m} x_{i}
$$

where $m$ is a positive integer. As in our first 

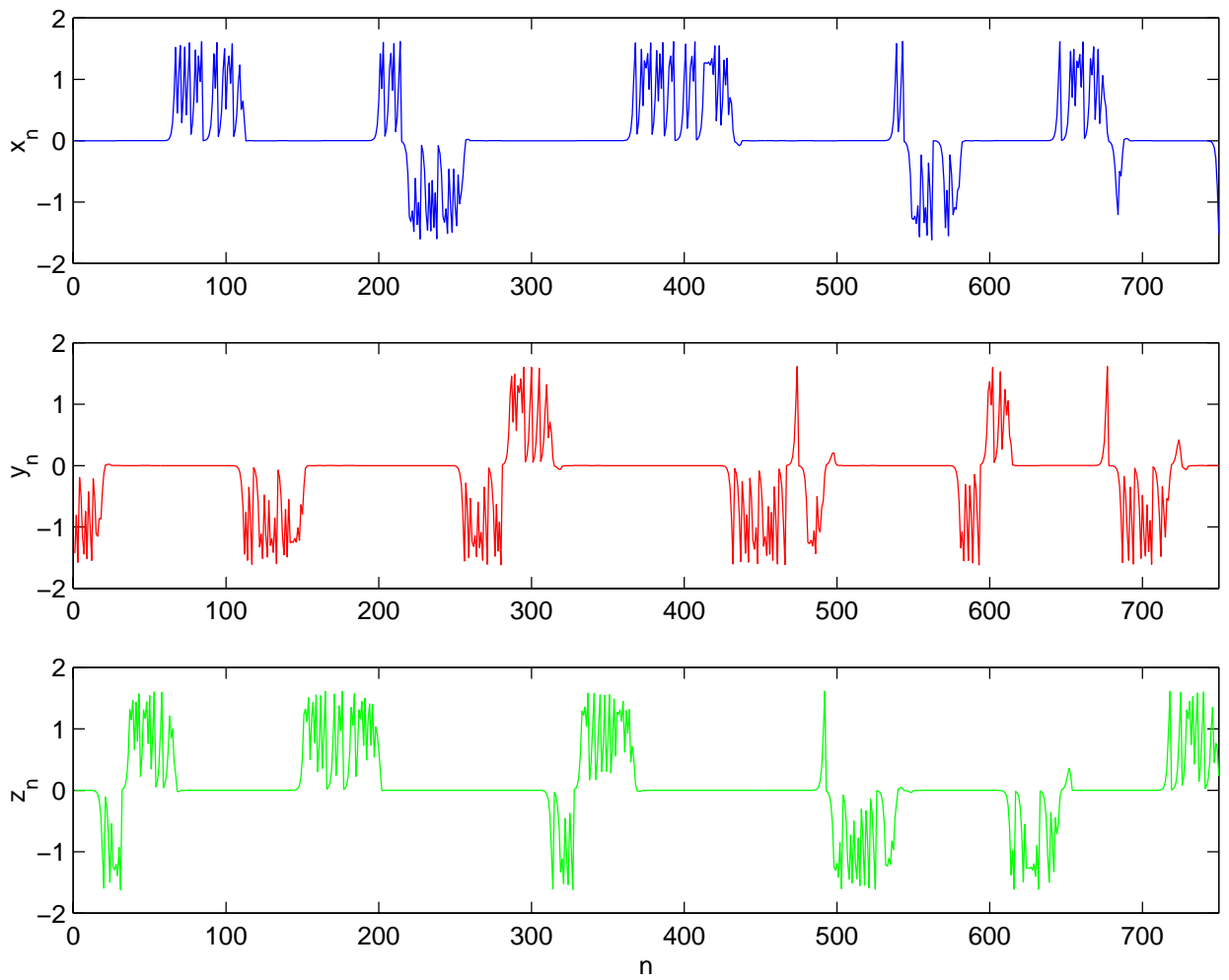

(a)
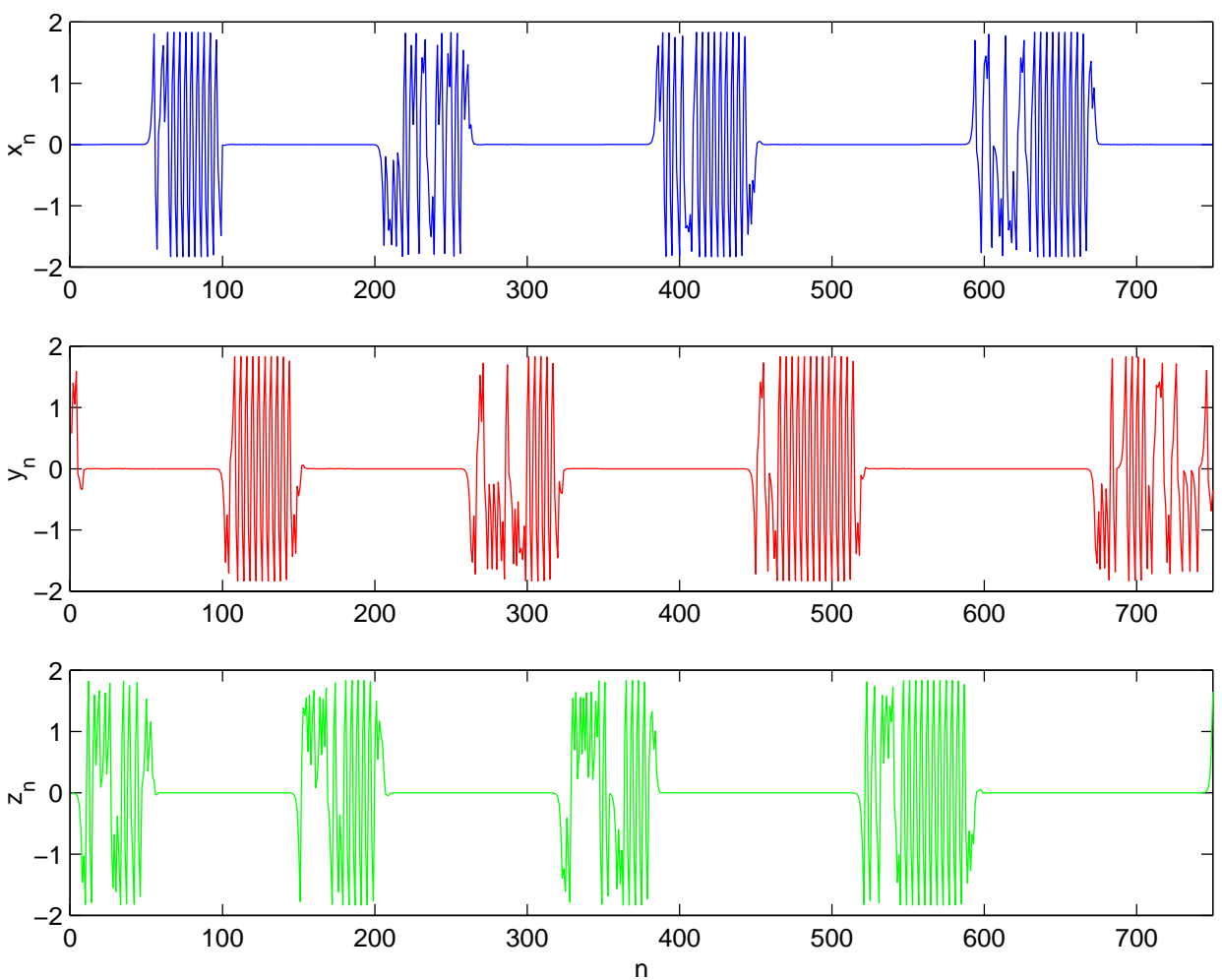

(b)

Fig. 7. Intermittent cycling chaos in the coupled cell system (4). (a) Near $\lambda_{c}$, with coupling strength $\alpha=-2.1$, switching between remnants of two chaotic attractors occurs frequently. (b) Away from $\lambda_{c}$, with coupling strength $\alpha=-2.2$, cycling chaos persists and the mergence of the two attractors is more uniform over $[0,2]$. 

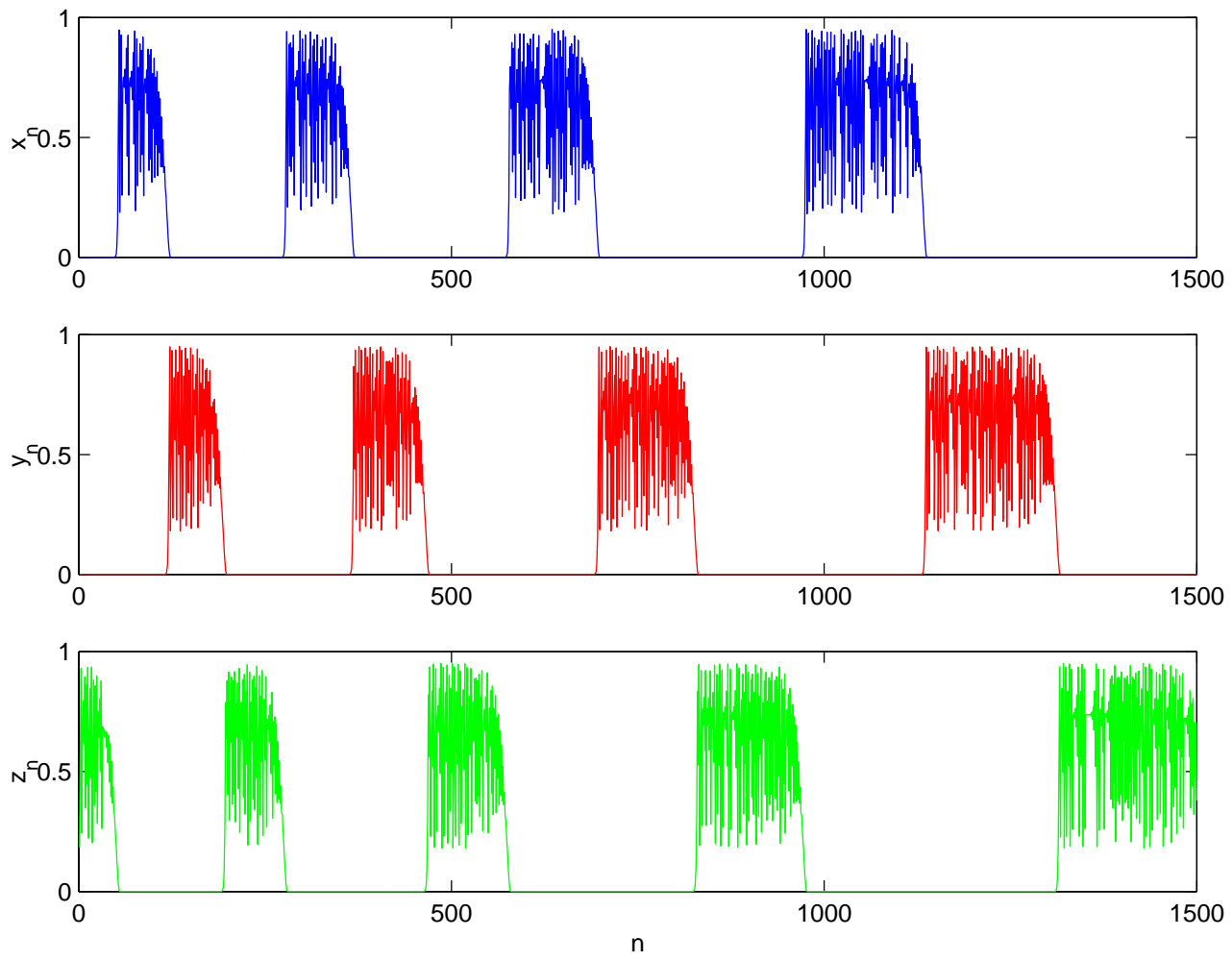

(a)
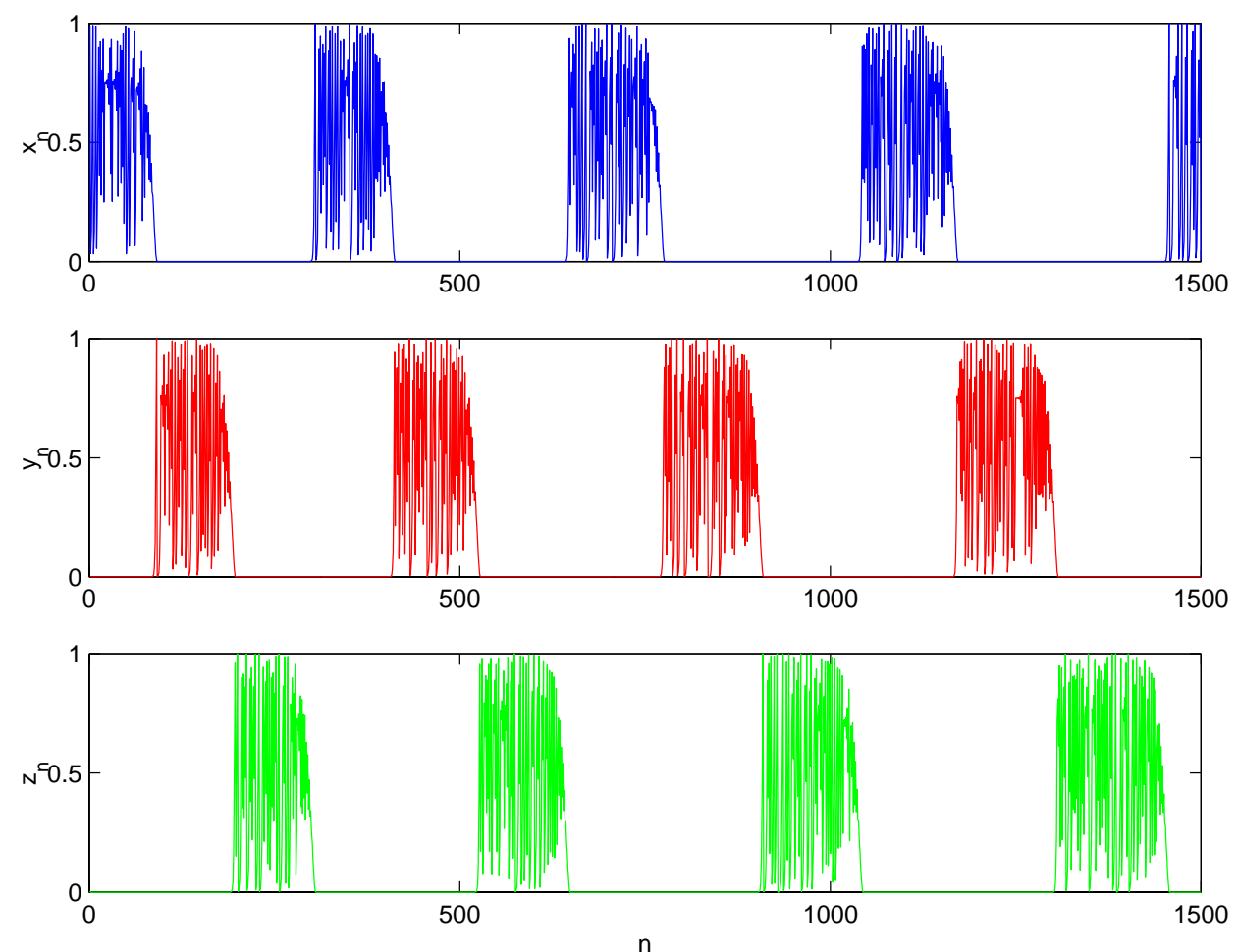

(b)

Fig. 8. Cycling chaos in a coupled cell system with internal cell dynamics governed by the logistic map $f(x, \lambda)=\lambda x(1-x)$. Initial conditions: $\left(x_{0}, y_{0}, z_{0}\right)=(0.0161,0.03,0.03)$. (a) Parameters: $m=10, \lambda=3.8$ and $\alpha=-3.72$. (b) Parameters: $m=10, \lambda=4$ and $\alpha=-3.97$. 
example, different combinations of parameter values (for $m, \lambda$, and coupling strength $\alpha$ ) yield cycling behavior between orbits of the internal dynamics of each cell. The wide variety of orbits in the logistic map, which now governs the internal dynamics of individual cells, also leads to a full range of cycles connecting fixed points with fixed points, periodic orbits with periodic orbits, and chaotic attractors with chaotic attractors. However, due to the lack of conjugate orbits, there are now only cycles of type I. For brevity purposes, we illustrate only, through Fig. 8, cycling chaos obtained at two different values of $\lambda$ where the logistic map is known to yield chaotic orbits. Observe that when $\lambda=3.8$, cell oscillations are confined to a subinterval of the interval $[0,1]$, while when $\lambda=4$, the oscillations fill the entire unit interval $[0,1]$. This is consistent with the chaotic attractors of the logistic map.

\section{Acknowledgments}

I would like to thank Prof. Marty Golubitsky for many stimulating conversations and for his mentorship in the area of bifurcation theory in systems with symmetry.

\section{References}

Armbruster, D., Guckenheimer, J. \& Holmes, P. [1988] "Heteroclinic cycles and modulated traveling waves in systems with $\mathbf{O}(2)$ symmetry," Physica D29, 257-282.
Buono, P. L., Golubitsky, M. \& Palacios, A. [2000] "Heteroclinic cycles in rings of coupled cells," Physica D143, 74-108.

Dellnitz, M., Field, M., Golubitsky, M., Ma, J. \& Hohmann, A. [1995] "Cycling chaos," Int. J. Bifurcation and Chaos 5(4), 1243-1247.

Dionne, B., Golubitsky, M. \& Stewart, I. [1996] "Coupled cells with internal symmetry. Part I: Wreath products," Nonlinearity 9, 559-574.

Field, M. J. [1980] "Equivariant dynamical systems," Trans. Amer. Math. Soc. 259(1), 185-205.

Guckenheimer, J. \& Holmes, P. [1988] "Structurally stable heteroclinic cycles," Math. Proc. Camb. Phil. Soc. 103, 189-192.

Manneville, P. [1990] Dissipative Structures and Weak Turbulence (Academic Press).

May, R. [1976] "Simple mathematical models with very complicated dynamics," Nature 261, 459-467.

Melbourne, I., Chossat, P. \& Golubitsky, M. [1989] "Heteroclinic cycles involving periodic solutions in mode interactions with $\mathbf{O}(2)$ symmetry," Proc. $R$. Soc. Edinburgh A113, 315-345.

Palacios, A. [2002] "Heteroclinic cycles in coupled systems of difference equations," J. Diff. Eq. Appl., to appear.

Rogers, T. \& Whitley, D. C. [1983] "Chaos in the cubic mapping," Math. Model. 4, 9-25. 